PROCEEDINGS OF THE

AMERICAN MATHEMATICAL SOCIETY

Volume 133, Number 10, Pages 2903-2909

S 0002-9939(05)07980-3

Article electronically published on April 25, 2005

\title{
A REMARK ON A CONJECTURE OF BORWEIN AND CHOI
}

\author{
ROBERT OSBURN
}

(Communicated by Wen-Ching Winnie Li)

\begin{abstract}
We prove the remaining case of a conjecture of Borwein and Choi concerning an estimate on the square of the number of solutions to $n=x^{2}+$ $N y^{2}$ for a squarefree integer $N$.
\end{abstract}

\section{INTRODUCTION}

We consider the positive definite quadratic form $Q(x, y)=x^{2}+N y^{2}$ for a squarefree integer $N$. Let $r_{2, N}(n)$ denote the number of solutions to $n=Q(x, y)$ (counting signs and order). In this note, we estimate

$$
\sum_{n \leq x} r_{2, N}(n)^{2}
$$

A positive squarefree integer $N$ is called solvable (or more classically "numerus idoneus") if $x^{2}+N y^{2}$ has one form per genus. Note that this means the class number of the form class group of discriminant $-4 N$ equals the number of genera, $2^{t}$, where $t$ is the number of distinct prime factors of $N$. Concerning $r_{2, N}(n)$, Borwein and Choi [1] proved the following:

Theorem 1.1. Let $N$ be a solvable squarefree integer. Let $x>1$ and $\epsilon>0$. We have

$$
\sum_{n \leq x} r_{2, N}(n)^{2}=\frac{3}{N}\left(\prod_{p \mid 2 N} \frac{2 p}{p+1}\right)(x \log x+\alpha(N) x)+O\left(N^{\frac{1}{4}+\epsilon} x^{\frac{3}{4}+\epsilon}\right),
$$

where the product is over all primes dividing $2 N$ and

$$
\alpha(N)=-1+2 \gamma+\sum_{p \mid 2 N} \frac{\log p}{p+1}+\frac{2 L^{\prime}\left(1, \chi_{-4 N}\right)}{L\left(1, \chi_{-4 N}\right)}-\frac{12}{\pi^{2}} \zeta^{\prime}(2),
$$

where $\gamma$ is the Euler-Mascheroni constant and $L\left(1, \chi_{-4 N}\right)$ is the L-function corresponding to the quadratic character mod $-4 N$.

Based on this result, Borwein and Choi posed the following:

Conjecture 1.2. For any squarefree $N$,

$$
\sum_{n \leq x} r_{2, N}(n)^{2} \sim \frac{3}{N}\left(\prod_{p \mid 2 N} \frac{2 p}{p+1}\right) x \log x .
$$

Received by the editors June 9, 2004.

2000 Mathematics Subject Classification. Primary 11E25, 11E45.

(C)2005 American Mathematical Society 
The main result in [10] is the following.

Theorem 1.3. Let $Q(x, y)=x^{2}+N y^{2}$ for a squarefree integer $N$ with $-N \not \equiv$ $1 \bmod 4$. Let $r_{2, N}(n)$ denote the number of solutions to $n=Q(x, y)$ (counting signs and order). Then

$$
\sum_{n \leq x} r_{2, N}(n)^{2} \sim \frac{3}{N}\left(\prod_{p \mid 2 N} \frac{2 p}{p+1}\right) x \log x .
$$

In this note, we settle the conjecture in the remaining case, namely

Theorem 1.4. For $-N \equiv 1 \bmod 4$, we have

$$
\sum_{n \leq x} r_{2, N}(n)^{2} \sim \frac{3}{N}\left(\prod_{p \mid 2 N} \frac{2 p}{p+1}\right) x \log x .
$$

\section{Preliminaries}

Let $Q(x, y)=a x^{2}+b x y+c y^{2}$ denote a positive definite integral quadratic form with discriminant $D=b^{2}-4 a c$ and $\operatorname{gcd}(a, b, c)=1$. Given $Q$, let $\kappa$ be the largest positive integer with $D / \kappa^{2}$ an integer congruent to 0 or 1 modulo 4 . We call $\kappa$ the conductor of $Q$ and set $d=D / \kappa^{2}$. Let $r(Q, n)$ be the number of representations of the integer $n$ by the form $Q$. We now relate $r(Q, n)$ to counting the number of integral ideals of norm $n$ in a given class in a generalized ideal class group.

Given $D=\kappa^{2} d$ we consider ideals in $\mathcal{O}_{K}$, where $K=\mathbb{Q}(\sqrt{d})$. Let $I_{\kappa}$ be the group of fractional ideals of $\mathcal{O}_{K}$ which are quotients of ideals coprime to $\kappa$ and let $P_{\kappa}$ be the subgroup of fractional ideals which are quotients of principal ideals $\langle\alpha\rangle \in I_{\kappa}$, where $\alpha \in \mathbb{Z}+\kappa \mathcal{O}$. Then set $C L_{\kappa}(K)=I_{\kappa} / P_{\kappa}$. The elements of $C L_{\kappa}(K)$ correspond bijectively to proper equivalence classes of positive definite quadratic forms of discriminant $D=\kappa^{2} d$. If the proper equivalence class of $Q$ corresponds to the ideal class $\mathfrak{c}$, then by [3], page 219, we have

$$
r(Q, n)=\sum_{r \mid \kappa} w\left((\kappa / r)^{2} d\right) J\left(\mathfrak{c}_{r}, n / r^{2}\right),
$$

where

$$
w(D)= \begin{cases}6 & \text { if } D=-3 \\ 4 & \text { if } D=-4 \\ 2 & \text { otherwise }\end{cases}
$$

Also $J\left(\mathfrak{c}_{r}, n\right)$ is the number of integral ideals of norm $n$ in the class $\mathfrak{c}_{r}$, where $\mathfrak{c}_{r}$ is the image of $\mathfrak{c}$ under the natural homomorphism $C L_{\kappa}(K) \rightarrow C L_{\kappa / r}(K)$. For the form $Q(x, y)=x^{2}+N y^{2}$ where $-N \equiv 1 \bmod 4$, the conductor $\kappa=2$ and so we have

$$
\begin{aligned}
r_{2, N}(n) & =w(-4 N) J(\mathfrak{c}, n)+w(-N) J\left(\mathfrak{c}_{2}, n / 4\right) \\
& =2 J(\mathfrak{c}, n)+w(-N) J\left(\mathfrak{c}_{2}, n / 4\right),
\end{aligned}
$$

where $\mathfrak{c}_{2}$ is the image under $C L_{2}(K) \rightarrow C L_{1}(K)$, that is, $\mathfrak{c}_{2}$ is a class in the ideal class group of $K=\mathbb{Q}(\sqrt{-N})$.

We now discuss a classical result of Rankin [11 and Selberg [12 which estimates the size of Fourier coefficients of a modular form. Specifically, if $f(z)=$ $\sum_{n=1}^{\infty} a(n) e^{2 \pi i n z}$ is a nonzero cusp form of weight $k$ on $\Gamma_{0}(N)$, then

$$
\sum_{n \leq x}|a(n)|^{2}=\alpha\langle f, f\rangle x^{k}+O\left(x^{k-\frac{2}{5}}\right),
$$


where $\alpha>0$ is an absolute constant and $\langle f, f\rangle$ is the Petersson scalar product. In particular, if $f$ is a cusp form of weight 1 , then $\sum_{n \leq x}|a(n)|^{2}=O(x)$. One can adapt their result to say the following. Given two cusp forms of weight $k$ on a suitable congruence subgroup of $\Gamma=S L_{2}(\mathbb{Z})$, say $f(z)=\sum_{n=1}^{\infty} a(n) e^{2 \pi i n z}$ and $g(z)=\sum_{n=1}^{\infty} b(n) e^{2 \pi i n z}$, then

$$
\sum_{n \leq x} a(n) \overline{b(n)} n^{1-k}=A x+O\left(x^{\frac{3}{5}}\right),
$$

where $A$ is a constant. In particular, if $f$ and $g$ are cusp forms of weight 1 , then $\sum_{n<x} a(n) \overline{b(n)}=O(x)$.

We conclude this section with a relationship between genus characters of generalized ideal class groups and the poles of the Rankin-Selberg convolution of Lfunctions. Recall that a group homomorphism $\chi: I_{2} \rightarrow S^{1}$ is an ideal class character if it is trivial on $P_{2}$, i.e.

$$
\chi(\langle a\rangle)=1
$$

for $a \equiv 1 \bmod \langle 2\rangle$. Thus an ideal class character is a character on the generalized class group $I_{2} / P_{2}$. Recall also that a genus character (see Chapter 12, section 5 in [5]) is an ideal class character of order at most two.

Let us also recall the notion of the Rankin-Selberg convolution of two L-functions. For squarefree $N$, consider two ideal class characters $\chi_{1}, \chi_{2}$ for $C L_{2}(K)$, the generalized ideal class group of $K=\mathbb{Q}(\sqrt{-N})$, and their associated Hecke L-series

$$
\begin{aligned}
& L_{2}\left(s, \chi_{1}\right)=\sum_{(\mathfrak{a}, 2)=1} \frac{\chi_{1}(\mathfrak{a})}{N(\mathfrak{a})^{s}}, \\
& L_{2}\left(s, \chi_{2}\right)=\sum_{(\mathfrak{a}, 2)=1} \frac{\chi_{2}(\mathfrak{a})}{N(\mathfrak{a})^{s}}
\end{aligned}
$$

which converge absolutely in some right half-plane. We form the convolution Lseries by multiplying the coefficients

$$
L_{2}\left(s, \chi_{1} \otimes \chi_{2}\right)=\sum_{(\mathfrak{a}, 2)=1} \frac{\chi_{1}(\mathfrak{a}) \chi_{2}(\mathfrak{a})}{N(\mathfrak{a})^{s}} .
$$

The following result describes a relationship between genus characters $\chi$ and the orders of poles of $L_{2}(s, \chi \otimes \chi)$. The proof is similar to that of Proposition 2.4 in [10].

Proposition 2.1. Let $\chi$ be an ideal class character for $C L_{2}(K),-N \equiv 1 \bmod 4$, and let $L_{2}(s, \chi)$ be the associated Hecke L-series. Then $\chi$ is a genus character if and only if $L_{2}(s, \chi \otimes \chi)$ has a double pole at $s=1$.

Remark 2.2. By Proposition 2.1, if $\chi$ is a non-genus character, then $L_{2}(s, \chi \otimes \chi)$ has at most a simple pole at $s=1$.

\section{Proof of Theorem 1.4}

Proof. As the proof is similar to that of Theorem 1.3 in [10, we sketch the relevant details. If $-N \equiv 1 \bmod 4$, then the discriminant of $K=\mathbb{Q}(\sqrt{-N})$ is $-N$. We also 
assume that $t$ is the number of distinct prime factors of $N$ and so the discriminant $-N$ also has $t$ distinct prime factors. For $K=\mathbb{Q}(\sqrt{-N})$, consider the zeta function

$$
\zeta_{K}(s, 2)=\sum_{(\mathfrak{a}, 2)=1} \frac{1}{N(\mathfrak{a})^{s}}
$$

where the sum is over those ideals $\mathfrak{a}$ of $\mathcal{O}_{K}$ prime to 2 . We now split up $\zeta_{K}(s, 2)$, according to the classes $\mathfrak{c}_{i}$ of the generalized ideal class group $C L_{2}(K)$, into the partial zeta functions (see page 161 of [7])

$$
\zeta_{\mathfrak{c}_{i}}(s)=\sum_{\mathfrak{a} \in \mathfrak{c}_{i}} \frac{1}{N(\mathfrak{a})^{s}}
$$

so that $\zeta_{K}(s, 2)=\sum_{i=0}^{h_{2}-1} \zeta_{\mathfrak{c}_{i}}(s)$, where $h_{2}$ is the order of $C L_{2}(K)$.

Let $\mathfrak{c}$ be the ideal class in $C L_{2}(K)$ which corresponds to the proper equivalence class of $Q(x, y)=x^{2}+N y^{2}$. Now let $\chi$ be an ideal class character of $C L_{2}(K)$ and consider the Hecke L-series for $\chi$, namely

$$
L_{2}(s, \chi)=\sum_{(\mathfrak{a}, 2)=1} \frac{\chi(\mathfrak{a})}{N(\mathfrak{a})^{s}} .
$$

We may now rewrite the Hecke L-series as

$$
L_{2}(s, \chi)=\sum_{i=0}^{h_{2}-1} \chi\left(\mathfrak{c}_{i}\right) \zeta_{\mathfrak{c}_{i}}(s) .
$$

Therefore, summing over all ideal class characters of $C L_{2}(K)$, we have

$$
\sum_{\chi} \bar{\chi}(\mathfrak{c}) L_{2}(s, \chi)=\sum_{i=0}^{h_{2}-1} \zeta_{\mathfrak{c}_{i}}(s)\left(\sum_{\chi} \bar{\chi}(\mathfrak{c}) \chi\left(\mathfrak{c}_{i}\right)\right) .
$$

The inner sum is non-zero precisely when $\mathfrak{c}=\mathfrak{c}_{i}$. Thus we have

$$
\zeta_{\mathfrak{c}}(s)=\frac{1}{h_{2}} \sum_{\chi} \bar{\chi}(\mathfrak{c}) L_{2}(s, \chi)
$$

and so

$$
\zeta_{\mathfrak{c}}(s)=\frac{1}{h_{2}}\left(L_{2}\left(s, \chi_{0}\right)+\overline{\chi_{1}}(\mathfrak{c}) L_{2}\left(s, \chi_{1}\right)+\cdots+\overline{\chi_{h_{2}-1}}(\mathfrak{c}) L_{2}\left(s, \chi_{h_{2}-1}\right)\right) .
$$

As $\chi_{0}$ is the trivial character, $L_{2}\left(s, \chi_{0}\right)=\zeta_{K}(s, 2)$. Comparing $n^{\text {th }}$ coefficients, we have

$$
J(\mathfrak{c}, n)=\frac{1}{h_{2}}\left(a_{n}+b_{1}(n)+\cdots+b_{h_{2}-1}(n)\right),
$$

where $a_{n}$ is the number of integral ideals of $\mathcal{O}_{K}$ prime to 2 and of norm $n$, and the $b_{i}$ 's are coefficients of weight 1 cusp forms (see [2]). Recall that we also have

$$
r_{2, N}(n)=2 J(\mathfrak{c}, n)+w(-N) J\left(\mathfrak{c}_{2}, n / 4\right)
$$

and so

$$
r_{2, N}(n)=\frac{2}{h_{2}}\left(a_{n}+b_{1}(n)+\cdots+b_{h_{2}-1}(n)\right)+w(-N) J\left(\mathfrak{c}_{2}, n / 4\right) .
$$


Thus

$$
\begin{aligned}
\sum_{n \leq x} r_{2, N}(n)^{2}=\frac{4}{h_{2}^{2}} & \left(\sum_{n \leq x} a_{n}^{2}+\sum_{\substack{i \\
n \leq x}} b_{i}(n)^{2}+2 \sum_{\substack{i \\
n \leq x}} a_{n} b_{i}(n)+\sum_{\substack{i \neq j \\
n \leq x}} b_{i}(n) b_{j}(n)\right) \\
& +\frac{4}{h_{2}} \sum_{n \leq x}\left(a_{n}+b_{1}(n)+\cdots+b_{h_{2}-1}(n)\right) w(-N) J\left(\mathfrak{c}_{2}, n / 4\right) \\
& +\sum_{n \leq x} w(-N)^{2} J\left(\mathfrak{c}_{2}, n / 4\right)^{2} .
\end{aligned}
$$

Assume $-N \equiv 1 \bmod 8$. Applying the main theorem in [6] to the Dirichlet series $\sum_{n=1}^{\infty} \frac{a_{n}^{2}}{n^{s}}$, we obtain

$$
\sum_{n \leq x} a_{n}^{2} \sim A x \log x
$$

where $A=\frac{1}{2 \pi^{2}} L\left(1, \chi_{-N}\right)^{2} \prod_{p \mid N} \frac{p}{p+1}$. As $-N$ has $t$ distinct prime factors, we have $2^{t}$ genus characters for $C L(K)$ where $K=\mathbb{Q}(\sqrt{-N})$. By [7] (see Theorem 1, page $127)$, we have $2^{t}$ genus characters for $C L_{2}(K)$. We now must estimate $\sum_{n \leq x} b_{i}(n)^{2}$. Let us now assume that the first $2^{t}-1$ terms arise from L-functions associated to genus characters. By Proposition 2.1 and an application of Perron's formula, we obtain

$$
\sum_{n \leq x} b_{i}(n)^{2} \sim A x \log x
$$

As this estimate holds for each $i$ such that $1 \leq i \leq 2^{t}-1$, the term $A x \log x$ appears $2^{t}$ times in the estimate of $\sum_{n \leq x} r_{2, N}(n)^{2}$. By Remark 2.2 and the Rankin-Selberg estimate, the remaining terms are all $O(x)$. Thus

$$
\sum_{n \leq x} r_{2, N}(n)^{2} \sim \frac{4}{h_{2}^{2}}\left(2^{t} \frac{1}{2 \pi^{2}} L\left(1, \chi_{-N}\right)^{2} \prod_{p \mid N} \frac{p}{p+1}\right) x \log x .
$$
Thus

By [4], we have $L\left(1, \chi_{-N}\right)=\frac{h \pi}{\sqrt{N}}$, where $h$ is the class number of $K$ and $h_{2}=h$.

$$
\sum_{n \leq x} r_{2, N}(n)^{2} \sim \frac{3}{N}\left(\prod_{p \mid 2 N} \frac{2 p}{p+1}\right) x \log x .
$$

For $-N \equiv 5 \bmod 8$, we have $h_{2}=3 h$ and again by $[\underline{6}$,

$$
\sum_{n \leq x} a_{n}^{2} \sim\left(\frac{9}{2 \pi^{2}} L\left(1, \chi_{-N}\right)^{2} \prod_{p \mid N} \frac{p}{p+1}\right) x \log x .
$$

Thus

$$
\sum_{n \leq x} r_{2, N}(n)^{2} \sim \frac{3}{N}\left(\prod_{p \mid 2 N} \frac{2 p}{p+1}\right) x \log x
$$

Remark 3.1. We would like to mention another approach which confirms Theorems 1.3 and 1.4. Let $Q \in \mathbb{Z}^{2 \times 2}$ be a non-singular symmetric matrix with even diagonal entries and $q(\mathbf{x})=\frac{1}{2} Q[\mathbf{x}]=\frac{1}{2} \mathbf{x}^{T} Q \mathbf{x}, \mathbf{x} \in \mathbb{Z}^{2}$, the associated quadratic form in two 
variables. Let $r(Q, n)$ denote the number of representations of $n$ by the quadratic form $Q$. Now consider the theta function

$$
\theta_{Q}(z)=\sum_{\mathbf{x} \in \mathbb{Z}^{2}} e^{\pi i z Q[\mathbf{x}]}
$$

The Dirichlet series associated with the automorphic form $\theta_{Q}$ is

$$
(4 \pi)^{-1 / 2} \zeta_{Q}\left(\frac{1}{2}+s\right)
$$

where

$$
\zeta_{Q}(s)=\sum_{n=1}^{\infty} \frac{r(Q, n)}{n^{s}}=\sum_{\mathbf{x} \in \mathbb{Z}^{2} \backslash\{\mathbf{0}\}} q(\mathbf{x})^{-s}
$$

for $\Re(s)>1$. A careful and involved application of the Rankin-Selberg method to the above Dirichlet series (see Theorems 2.1 and 5.1 in [8] and Theorem 5.2 in [9]) combined with a Tauberian argument yields the following (see Theorem 6.1 in [8]):

$$
\sum_{n \leq x} r(Q, n)^{2} \sim A_{Q} x \log x
$$

where

$$
A_{Q}=12 \frac{A(q)}{q} \prod_{p \mid q}\left(1+\frac{1}{p}\right)^{-1} .
$$

Here $q=\operatorname{det} Q$ and $A(q)$ denotes the multiplicative function defined by

$$
A\left(p^{e}\right)=2+\left(1-\frac{1}{p}\right)(e-1),
$$

where $p$ is an odd prime, $e \geq 1$, and

$$
A\left(2^{e}\right)=\left\{\begin{array}{l}
1 \quad \text { if } e \leq 1 \\
2 \text { if } e=2, \\
e-1 \text { if } e \geq 3 .
\end{array}\right.
$$

Let us now turn to our situation. Consider $q(\mathbf{x})=x^{2}+N y^{2}=\frac{1}{2} \mathbf{x}^{T} Q \mathbf{x}$, where $Q=\left(\begin{array}{cc}2 & 0 \\ 0 & 2\end{array}\right), N$ squarefree. Thus $q=4 N$. Suppose $N$ has $t$ distinct prime factors. Then $A(4 N)=2^{t+1}$ and so

$$
A_{Q}=\frac{3}{N} 2^{t+1} \prod_{p \mid 2 N}\left(1+\frac{1}{p}\right)^{-1}=\frac{3}{N} \prod_{p \mid 2 N} \frac{2 p}{p+1} .
$$

\section{ACKNOWLEDGMENTS}

The author thanks Ram Murty for many productive discussions and for his comments on a preliminary version of this paper.

\section{REFERENCES}

[1] J. Borwein, K.K. Choi, On Dirichlet Series for sums of squares, Rankin memorial issues, Ramanujan J. 7 (2003), no.1-3, 95-127. MR2035795

[2] D. Bump, Automorphic forms and representations, Cambridge Studies in Advanced Mathematics, 55, Cambridge University Press, 1997. MR1431508 (97k:11080)

[3] R. Chapman, A. van der Poorten, Binary Quadratic Forms and the Eta Function, Number theory for the millennium, I (Urbana, IL, 2000), 215-227, A K Peters, Natick, MA, 2002. MR:1956227 (2004b:11045)

[4] H. Cohn, Advanced Number Theory, Dover Publications, Inc., New York, 1980. MR0594936 (82b:12001) 
[5] H. Iwaniec, Topics in Classical Automorphic Forms, Graduate Studies in Mathematics, Vol. 17, Amer. Math. Soc., Providence, RI, 1997. MR,1474964 (98e:11051)

[6] M. Kühleitner, W.G. Nowak, The average number of solutions to the Diophantine equation $U^{2}+V^{2}=W^{3}$ and related arithmetic functions, Acta Math. Hungar. 104 (2004), 225-240. MR 2075922

[7] S. Lang, Algebraic Number Theory, Second Edition, Springer-Verlag, New York, 1994. MR.1282723 (95f:11085)

[8] W. Müller, The mean square of Dirichlet series associated with automorphic forms, Monatsh. Math. 113 (1992), 121-159. MR1154475 (93g:11050)

[9] W. Müller, The Rankin-Selberg Method for non-holmorphic automorphic forms, J. Number Theory 51 (1995), 48-86. MR1321724 (95m:11049)

[10] R. Murty, R. Osburn, Representations of integers by certain positive definite binary quadratic forms, Ramanujan J., to appear.

[11] R.A. Rankin, Contributions to the theory of Ramanujan's function $\tau(n)$ and similar functions. II. The order of the Fourier coefficients of integral modular forms, Proc. Cambridge Philos. Soc. 35 (1939), 357-373. MR0000411 (1:69d)

[12] A. Selberg, Bemerkungen über eine Dirichletsche Reihe, die mit der Theorie der Modulformen nahe verbunden ist, Archiv. Math. Natur. B 43 (1940), 47-50. MR0002626 (2:88a)

Department of Mathematics and Statistics, Queen's University, Kingston, Ontario, CANADA K7L 3N6

E-mail address: osburnr@mast.queensu.ca

Current address: Max-Planck Institut für Mathematik, Vivatsgasse 7, Bonn, Germany

E-mail address: osburn@mpim-bonn.mpg.de 